\title{
Automatic Finger Interruption Detection in Electroluminescence Images of Multicrystalline Solar Cells
}

\author{
Din-Chang Tseng, ${ }^{1}$ Yu-Shuo Liu, ${ }^{1}$ and Chang-Min Chou ${ }^{2}$ \\ ${ }^{1}$ Department of Computer Science and Information Engineering, National Central University, No. 300, Jhongda Road, \\ Jhongli 32001, Taiwan \\ ${ }^{2}$ Department of Electronic Engineering, Chien Hsin University of Science and Technology, No. 229, Jianxing Road, \\ Jhongli 32097, Taiwan
}

Correspondence should be addressed to Chang-Min Chou; changmin@uch.edu.tw

Received 12 December 2014; Accepted 11 February 2015

Academic Editor: Mo Li

Copyright (C) 2015 Din-Chang Tseng et al. This is an open access article distributed under the Creative Commons Attribution License, which permits unrestricted use, distribution, and reproduction in any medium, provided the original work is properly cited.

\begin{abstract}
This study provides an automatic method for detecting finger interruptions in electroluminescence $(E L)$ images of multicrystalline solar cells. The proposed method is a supervised classification method. We obtain regions of interest (ROI) by separating the $E L$ image to several regions. The fingers within each $R O I$ are candidates for defect detection. We horizontally scan each $R O I$ region and extract features from each finger pixel. In the training stage, we record a set of features which are extracted from interrupted fingers and noninterrupted fingers. These features are represented as points in a spectral embedding space produced by spectral clustering method. These points will be classified into two clusters: interrupted fingers and noninterrupted fingers. In the classification stage, we firstly detect the position of fingers in an EL image and obtain features from each finger. The set of features in each finger combined with known features in the training stage will be represented as points in the spectral embedding space and then will be classified to the cluster with nearer cluster centroid of known features. Experimental results show that the proposed method can effectively detect finger interruptions on a set of $E L$ images of various solar cells.
\end{abstract}

\section{Introduction}

A solar cell is an electrical device that converts the sunlight directly into electricity by the photovoltaic effect. By far, the most prevalent bulk material for solar cells is crystalline silicon because of the competitive conversion efficiency and usable lifespan. Crystalline silicon solar cells can be in the form of monocrystalline and multicrystalline cells. Multicrystalline solar cells are more common than monocrystalline solar cells due to lower material and manufacturing costs [1]. Finished multicrystalline silicon solar cells are frequently found to be defective. Defects can be divided into two categories [2]: intrinsic and extrinsic defects. Intrinsic defects consist of grain boundaries and dislocations, which will decrease the photoelectric conversion efficiency. Extrinsic defects consist of microcracks, breaks, and finger interruptions, which will shorten lifetime, reduce efficiency, and make authentication fails of cells $[3,4]$.
The electroluminescence (EL) imaging technique has been proposed in recent years to highlight the deficiencies that degrade the conversion efficiency of a solar cell [5]. An example of EL image of a multicrystalline silicon solar cell is shown in Figure 1(a). A schematic of defects in Figure 1(a) is shown in Figure 1(b). In Figure 1(b), the region within the white dashed rectangle is a solar cell, the vertical black thin strips are fingers, and the two horizontal thick strips are busbars (sometimes there are more than two bus-bars). The black regions other than fingers and bus-bars are defects, where the black thin strip marked as defect 1 is a microcrack, the black stripes marked as defect 2 are finger interruptions, and other irregular black regions are internal defects.

Automatic extrinsic defect detection in EL images of multicrystalline silicon solar cell is usually very difficult due to the disturbances of intrinsic defects. In recent years, automatic detection of microcracks has been explored in some literatures [6-9]. These researches usually detect defects 


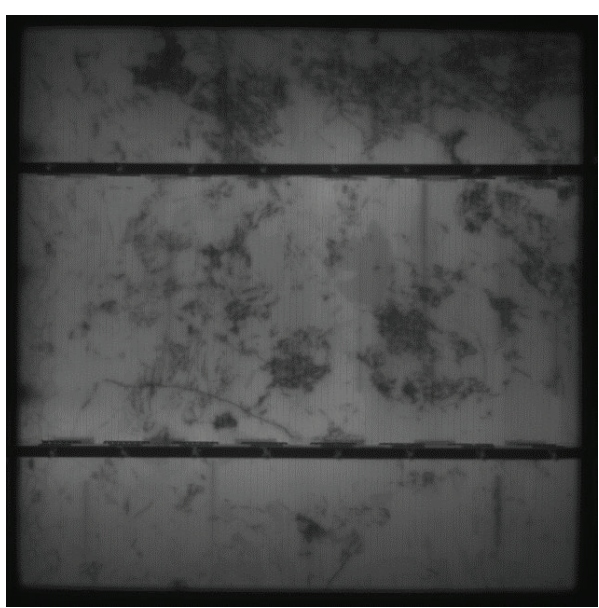

(a)

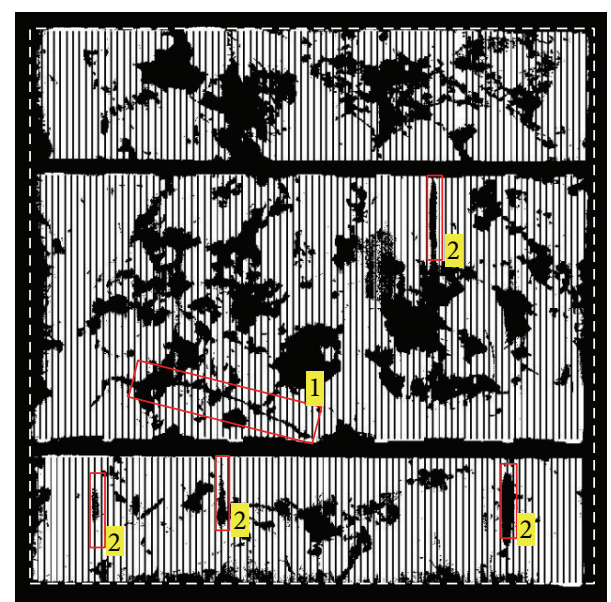

(b)

Figure 1: Defects in an EL image of multicrystalline silicon solar cell. (a) An EL image. (b) A schematic of defects, where defect 1 is microcrack and defects 2 are interrupted fingers.

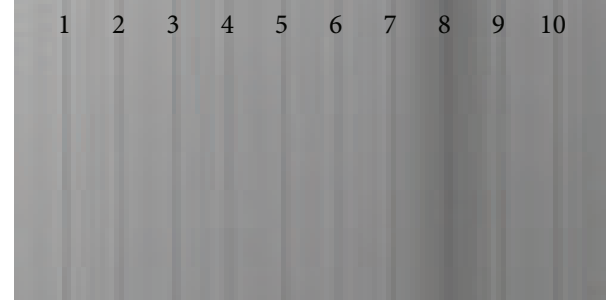

Figure 2: An example of interrupted fingers and noninterrupted fingers. These numerics are the number of fingers and the 8 th finger is an interrupted finger defect.

by using gray level and shape differences. However, these approaches may fail in detecting finger interruption when the background is seriously interfered and the contrast is low. Tsai et al. [6] proposed a Fourier image reconstruction scheme to detect microcracks and finger interruptions in multicrystalline silicon solar cells. Their method is based on the fact that the defects in the solar cell appear as line or bar shaped objects and those appears are darker than its surroundings in the EL image. In their method, correct implementation depends crucially on the setting of threshold values for image reconstruction. In most cases, the threshold value has to be determined interactively through trail-anderror method.

In an EL image, finger interruptions are shown as dark stripes on both sides of the fingers, while noninterrupted fingers are lighting on both sides of the fingers. An example of interrupted fingers and noninterrupted fingers is shown in Figure 2. In this study, we extract the variation of gray level on both sides of a finger as its features to resist the problems of background interference and unobvious defects.

The remainder of this paper is organized as follows. In Section 2, we review the principle of spectral clustering algorithm. Section 3 explains the proposed defect detection method in detail. The experiment results are presented in
Section 4. Finally, concluding remarks and future works are discussed in Section 5.

\section{Spectral Clustering Algorithm}

We use spectral clustering method to cluster features of fingers. In our experiments, the features of fingers are not linearly separable. The $k$-mean method works fine in clustering linear separable samples only. Spectral clustering method has the advantage of using graph cuts as objective functions for nonlinear data separation [10]. Spectral clustering algorithm refers to a class of techniques which rely on the eigenstructure of a similarity matrix to partition samples into disjoint clusters with samples in the same cluster having high similarity and samples in different clusters having low similarity. This algorithm makes use of the spectral (eigenvalues) of the similarity matrix to perform dimensionality reduction for clustering objects in fewer dimensions (embedding space). Therefore, spectral clustering method has been widely used in several areas such as information retrieval and computer vision [11-14].

Given a dataset $\mathbf{X}=\left\{\mathbf{x}_{1}, \mathbf{x}_{2}, \ldots, \mathbf{x}_{n}\right\}$, the spectral clustering algorithm constructs a similarity matrix $\mathbf{S}^{n \times n}$, where $\mathbf{s}_{i j} \geq$ 0 reflects the relationship between $\mathbf{x}_{i}$ and $\mathbf{x}_{j}$. It then uses the similarity information to group elements in $\mathbf{X}$ into $k$ clusters. There are several variants of spectral clustering. Here, we consider the commonly used normalized spectral clustering. A similarity function is defined as a Gaussian distribution:

$$
s_{i j}= \begin{cases}e^{\left(-\left\|\mathbf{x}_{i}-\mathbf{x}_{j}\right\|^{2} / 2 \sigma^{2}\right)}, & \text { if } i \neq j, \\ 0, & \text { if } i=j,\end{cases}
$$

where $\sigma$ is a scaling parameter to control how rapidly the similarity $s_{i j}$ reduces with the distance between $\mathbf{x}_{i}$ and $\mathbf{x}_{j}$. Consider the Laplacian matrix:

$$
\mathbf{L}=\mathbf{D}-\mathbf{S},
$$




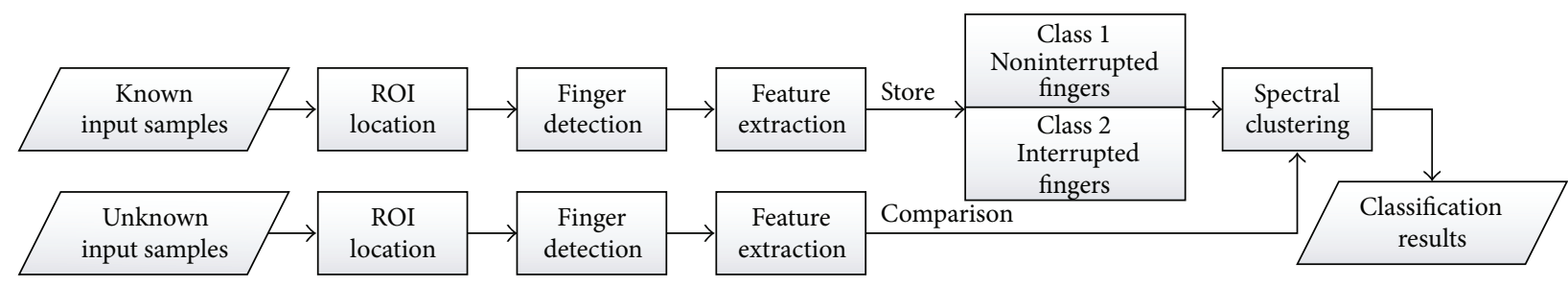

FIGURE 3: Block diagram of the proposed method.

where $\mathbf{D}$ is a diagonal matrix defined as

$$
\begin{array}{r}
d_{i j}= \begin{cases}\sum_{j=1}^{n} s_{i j}, & \text { if } i \neq j \\
0, & \text { if } i=j\end{cases} \\
i=1, \ldots, n .
\end{array}
$$

The Laplacian matrix $\mathbf{L}$ is symmetric and positive semidefinite. Compute eigenvalues $\lambda$ and eigenvectors $\mathbf{y}$ for the generalized eigenvector problem:

$$
\mathbf{L y}=\lambda \mathbf{D y} \text {. }
$$

Let eigenvector set $\mathbf{Y}^{n \times k}=\left\{\mathbf{y}_{0}^{i}, \ldots, \mathbf{y}_{k-1}^{i}\right\}, i=1 \cdots n$, be the solutions of (4). Eigenvector $\mathbf{y}^{i}$ represents data $\mathbf{x}_{i}$ in the embedding space and is ordered according to its eigenvalues with $\mathbf{y}_{0}^{i}$ representing the smallest eigenvalue.

\section{The Proposed Method}

The block diagram of the proposed method is shown in Figure 3. We obtain regions of interest (ROI) by separating the EL image to several regions. The fingers within each ROI are candidates for defect detection. We horizontally scan each ROI region and extract features from each finger pixel. In the training stage, we record a set of features which are extracted from interrupted fingers and noninterrupted fingers. These features are represented as points in a spectral embedding space produced by spectral clustering method. These points will be classified into two clusters: interrupted fingers and noninterrupted fingers. In the classification stage, we firstly detect the position of fingers in an EL image and obtain features from each finger. The set of features in each finger combined with known features in the training stage will be represented as points in the spectral embedding space and then be classified to the cluster with nearer cluster centroid of known features.

3.1. ROI Location. In an industrial production line, the production of solar cells is under precise control to follow its specifications. As a result, all produced solar cells have the same size, bus-bar positions, and width of bus-bars in the EL images. An example of EL image with size $m \times n$ is shown in Figure 4. In Figure 4, bus-bars are shown as horizontal black bold strips; ROIs are regions between bus-bars. We use slide windows to locate positions of ROIs by horizontal

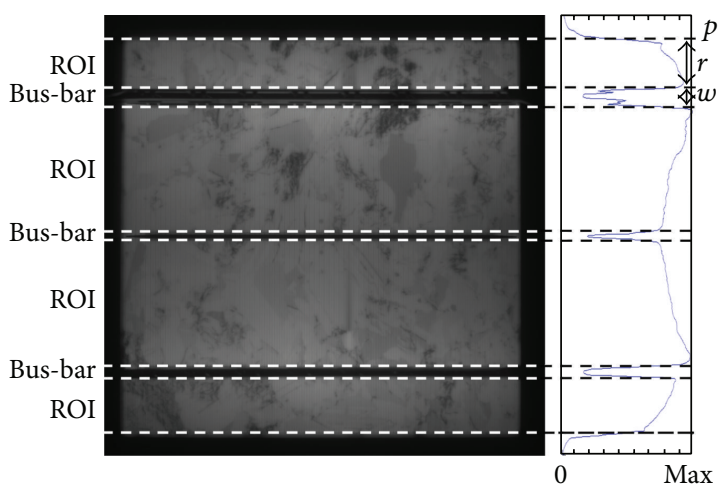

(a)

(b)

FIgUre 4: The schematic of separating ROIs in an EL image. (a) An EL image. (b) The horizontal projections of EL image.

projections. Let $I$ be an EL image and $I(x, y)$ the gray level of pixel $(x, y)$. The horizontal projections $f_{h}(y)$ are defined as

$$
f_{h}(y)=\sum_{i=1}^{m} I(i, y), \quad y=1, \ldots, n .
$$

Since the bus-bars have lower gray level, we propose to find the top position $p$ of the top ROI by accumulating the horizontal projection in a slide window of width $w$,

$$
p=\underset{d}{\arg }\left\{\min _{1 \leq d \leq n-t} \sum_{j=1}^{w} f_{h}(r+j+d)\right\},
$$

where $w$ is set to be the height of the top bus-bar and $r$ is the height of the top ROI, as shown in Figure 4. In a production line, the values of $r$ and $w$ are preknown. After finding $p$, the locations of ROIs can be easily calculated according to the specification information of bus-bar heights and ROI heights.

3.2. Finger Detection. Now, the positions of fingers should be detected from each ROI. Fingers are vertical black thin strips. The vertical projections $f_{v}(x)$ of each ROI can be calculated by

$$
f_{v}(x)=\sum_{i=1}^{\text {Height }_{\mathrm{ROI}}} I(x, i), \quad x=1, \ldots, m,
$$

where Height $_{\mathrm{ROI}}$ is the height of ROI and $m$ is the width of ROI. The second derivative $\nabla^{2} f_{v}(x)$ is calculated by

$$
\nabla^{2} f_{v}(x)=f_{v}(x+1)+f_{v}(x-1)-2 f_{v}(x) .
$$




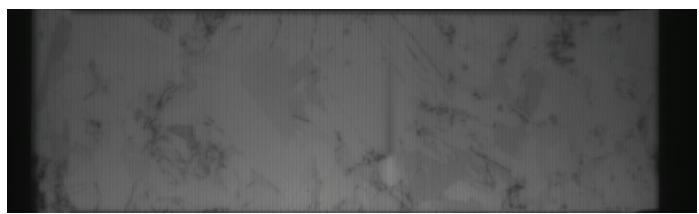

(a)

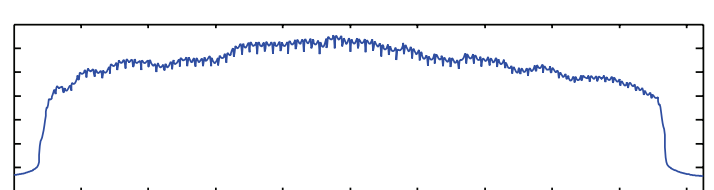

(b)

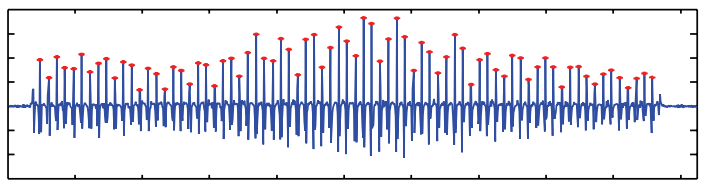

(c)

FIGURE 5: The ROI image and its vertical projections. (a) The ROI image. (b) The vertical projections of ROI image. (c) The second derivative of vertical projections. The star marks show the positions of fingers.

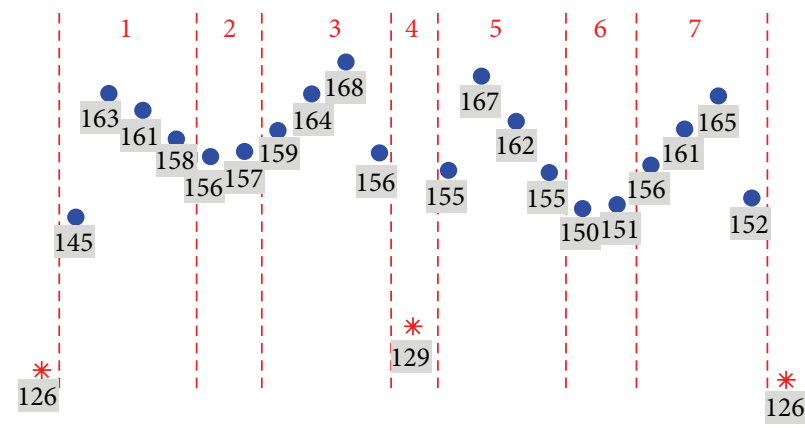

(a)

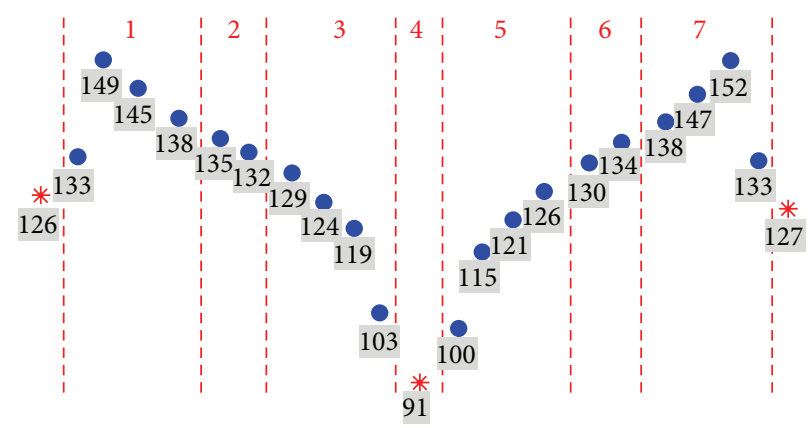

(b)

Figure 6: A typical horizontal line of a finger pixel. (a) A noninterrupted finger pixel. (b) An interrupted finger pixel. The value shown under each black dot represents the gray level of that pixel.

The positions of fingers can he identified by finding local maximums of $\nabla^{2} f_{v}(x)$. An example of finding the fingers is shown in Figure 5. An image of ROI is shown in Figure 5(a); the vertical projections are shown in Figure 5(b); the second derivatives of vertical projections are shown in Figure 5(c); the star marks show the positions of fingers. The number of fingers in a ROI image is preknown as $n$. Since there will be more than $n$ local maximums in the second derivative, we need to figure out the correct $n$ maximums which represent the $n$ fingers in the ROI. In our proposed method, the $n$ consecutive maximums with largest sum will represent the correct positions of $n$ fingers.

3.3. Feature Extraction. After the positions of fingers are detected, we horizontally scan each ROI and extract features for each finger. An interrupted finger has dark stripes on both sides, while a normal noninterrupted finger is lighting on both sides. Thus, for a noninterrupted finger, the pixels right beside the finger should have higher gray levels; the pixels in the middle of two neighboring fingers should have lower gray levels; the finger itself should have the lowest gray level. A typical horizontal line of a noninterrupted finger is shown in Figure 6(a). A typical horizontal line of an interrupted finger is shown in Figure 6(b). The value shown under each black dot represents the gray level of that pixel.
According to the difference between noninterrupted fingers and interrupted fingers, we propose to extract seven features for each finger pixel: three from the left side and three from the right side and one is the gray level of the finger pixel itself. For each side, we first divide the pixels between the finger and its neighboring finger into three partitions: a middle partition, a left partition, and a right partition. If the number of these pixels is odd, the middle partition consists of three pixels in central; otherwise it consists of two pixels. The left and right partitions consist of the other pixels in the left and right sides of the middle partition, respectively. For the middle partition, we take the average gray level as a feature. For the left and right partitions, we take their maximum gray levels as features. For example, the seven features are 126, 163, 156.5, 168, 129, 167, 150.5, 165, and 126 in Figure 6(a) and 126, $149,133.5,129,91,126,132,152$, and 127 in Figure 6(b).

3.4. Finger Classification. In the training stage, we manually obtain features of known noninterrupted and interrupted fingers and give cluster labels to these features, respectively. We apply the spectral clustering algorithm to transfer these labeled features to an embedding space and assign these features into two clusters: a noninterrupted cluster and an interrupted cluster, according to their labels. In the classification stage, we obtain a set of features from pixels of 


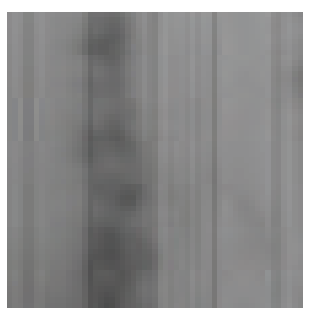

(a1)

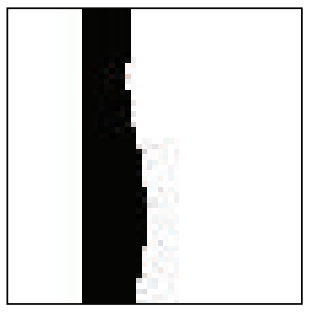

(b1)

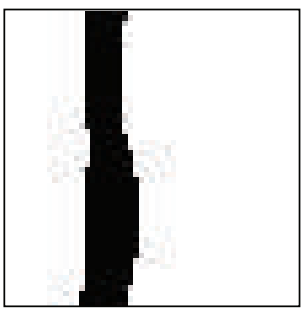

(c1)

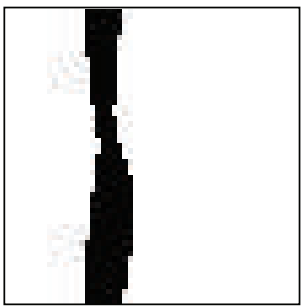

(d1)

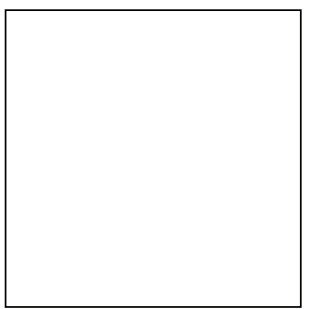

(e1)

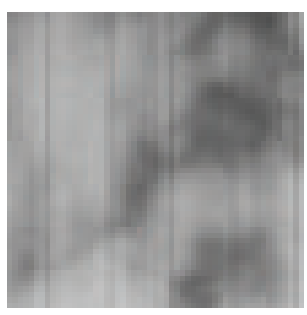

(a2)

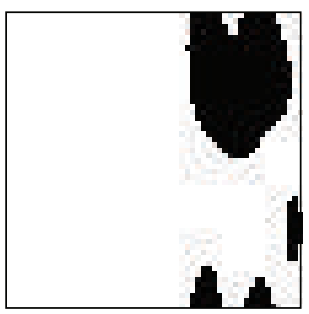

(b2)

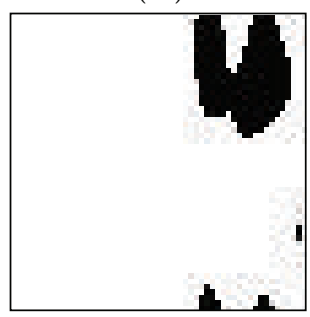

(c2)

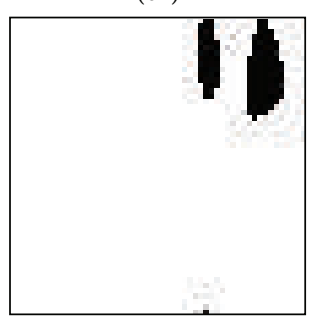

(d2)

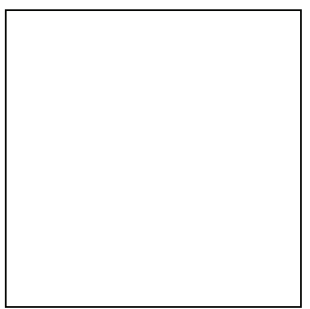

(e2)

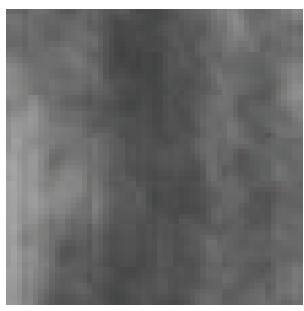

(a3)

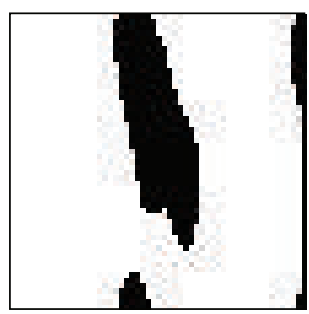

(b3)

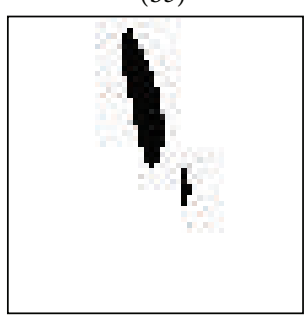

(c3)

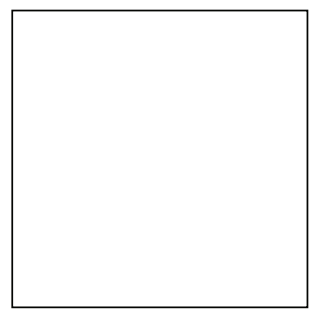

(d3)

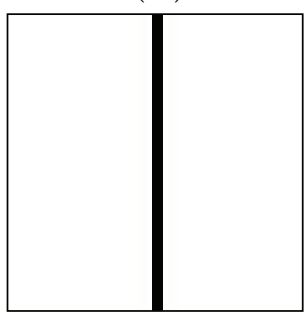

(e3)

Figure 7: The experimental results of EL subimages with noninterrupted finger defects on a $55 \times 55$ subimage. (a1)-(a3) The EL subimages. (b1)-(b3) The results of FIR method with $K_{\Delta f}=0.5$. (c1)-(c3) The results of FIR method with $K_{\Delta f}=1$. (d1)-(d3) The results of FIR method with $K_{\Delta f}=1.5$. (e1)-(e3) The results of our proposed method. The black regions and lines indicate detected defects.

an unknown finger. The classification steps are described as follows.

(1) Combine the feature set of the unknown finger with known features in the training stage.

(2) Each feature set $\mathbf{f}=\left\{f_{1}, f_{2}, f_{3}, f_{4}, f_{5}, f_{6}, f_{7}\right\}$ is used to construct a data set $\mathbf{x}=\left\{x_{1}, x_{2}, x_{3}, x_{4}, x_{5}, x_{6}\right\}$, where $x_{i}=f_{i}-f_{i+1}, i=1,2, \ldots, 6$, for applying to the spectral clustering algorithm.
(3) For all data sets, apply (1) to construct a similarity matrix $S^{(n+t) \times(n+t)}$, where $n$ is the number of known features in the training stage and $t$ is the number of pixels in the unclassified finger.

(4) Apply (2) to compute the Laplacian matrix $\mathbf{L}$ of $\mathbf{S}$.

(5) Apply (4) to compute the first $k$ largest eigenvectors of $\mathbf{L}$; and construct $\mathbf{Y}^{(n+t) \times k}$. 


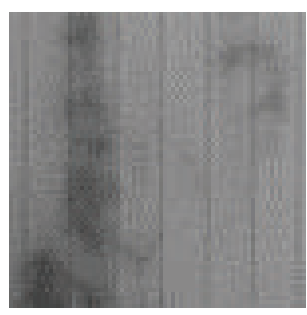

(a1)

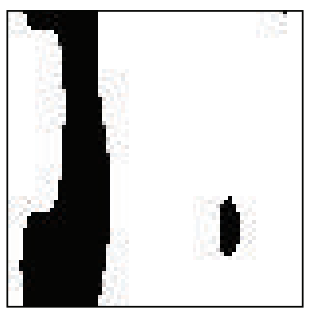

(b1)

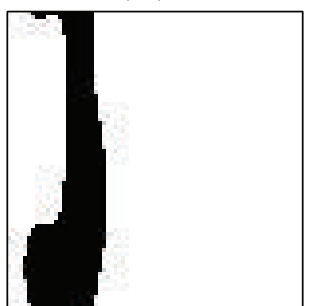

(c1)

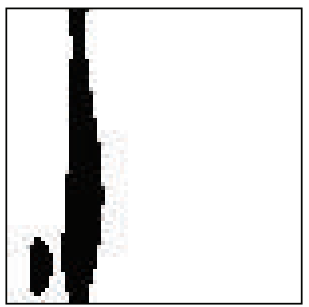

(d1)

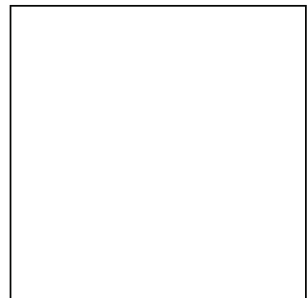

(e1)

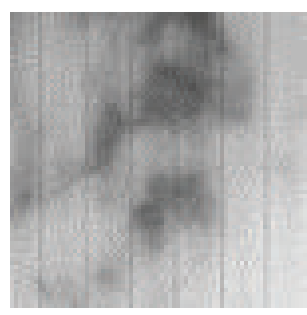

(a2)

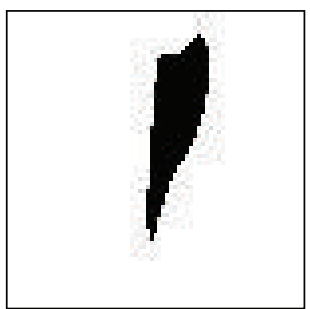

(b2)

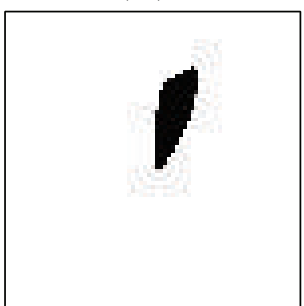

(c2)

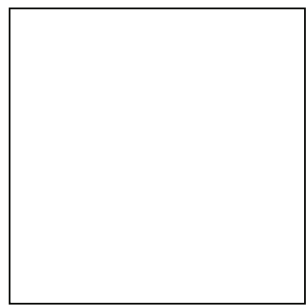

(d2)

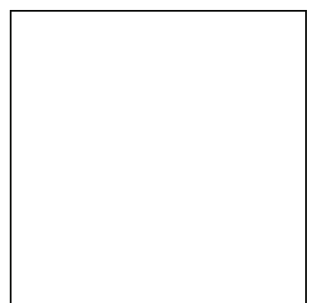

(e2)

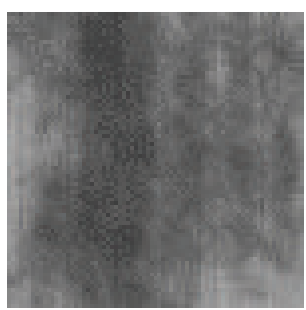

(a3)

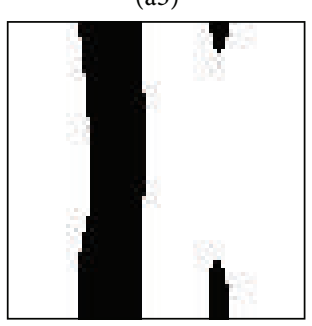

(b3)

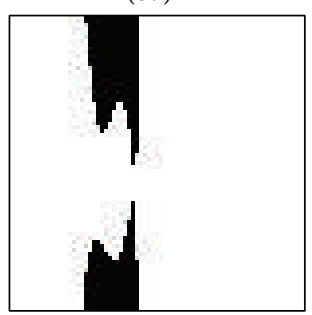

(c3)

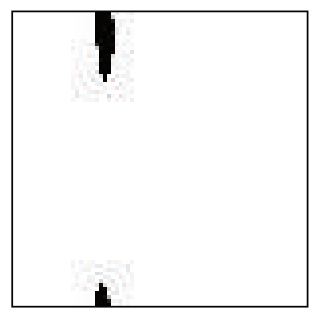

$(\mathrm{d} 3)$

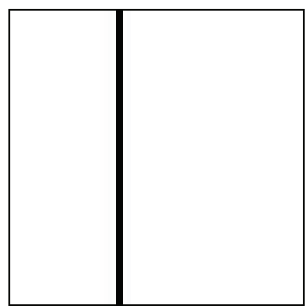

(e3)

FIGURE 8: The experimental results of EL subimages with noninterrupted finger defects on a $75 \times 75$ subimage. (al)-(a3) The EL subimages. (b1)-(b3) The results of FIR method with $K_{\Delta f}=0.5$. (c1)-(c3) The results of FIR method with $K_{\Delta f}=1$. (d1)-(d3) The results of FIR method with $K_{\Delta f}=1.5$. (e1)-(e3) The results of our proposed method. The black regions and lines indicate detected defects.

(6) The $n+t$ rows in $\mathbf{Y}^{(n+t) \times k}$ represent $n+t$ points in the embedding space $\mathbf{R}$. The first $n$ points represent the $n$ known features in the training stage. They are labeled as noninterrupted or interrupted cluster. The remaining $t$ points will be classified to the cluster with nearer cluster centroid of known features.

A finger contains numbers of finger pixels. If the number of finger pixels belonging to the interrupted cluster is larger than a predefined ratio, the finger will be classified as an interrupted finger.

\section{Experiments}

In this section, we present the experimental results from 60 multicrystalline solar cells with various defect types in EL images. Each solar cell has two or three bus-bars with 72 or 82 fingers in each ROI. The size of each solar cell is 


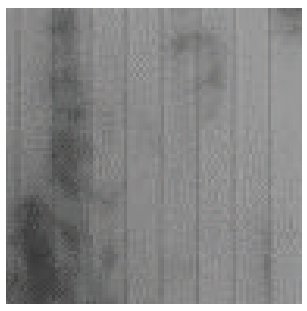

(a1)

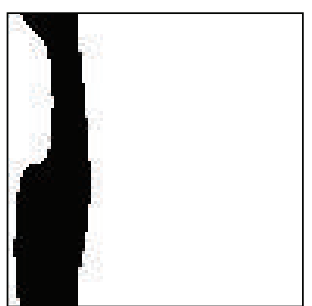

(b1)

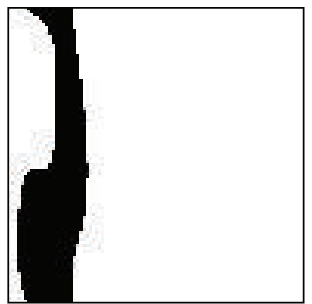

(c1)

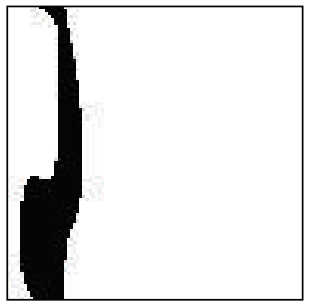

(d1)

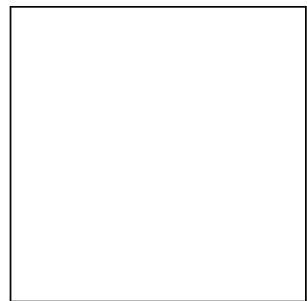

(e1)

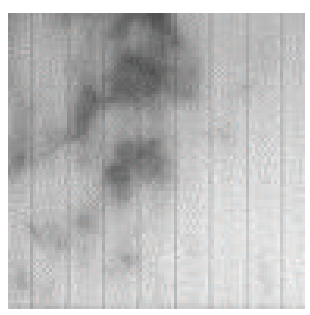

(a2)

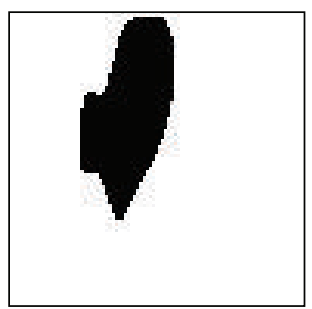

(b2)

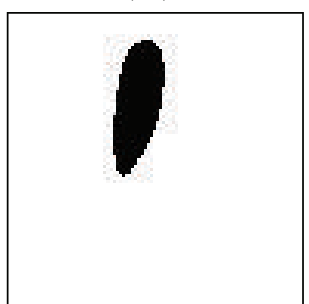

(c2)

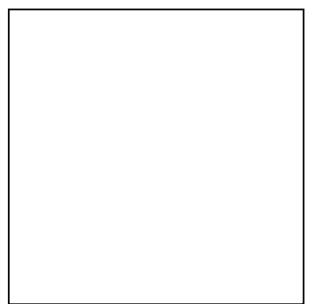

(d2)

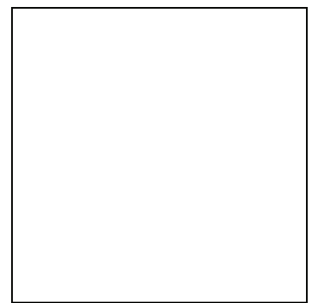

(e2)

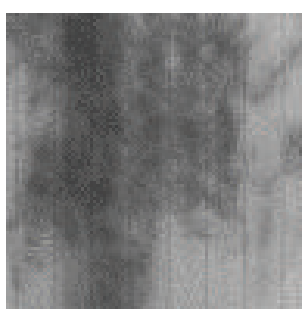

(a3)

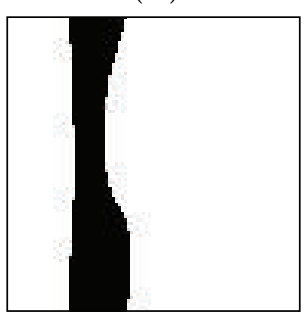

(b3)

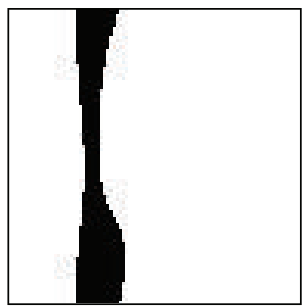

(c3)

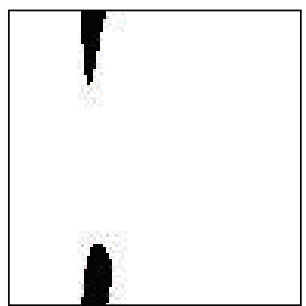

$(\mathrm{d} 3)$

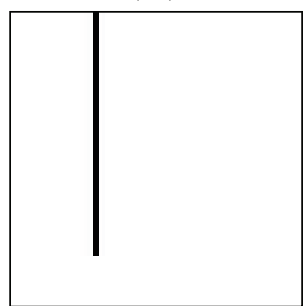

(e3)

Figure 9: The experimental results of EL subimages with noninterrupted finger defects on a $95 \times 95$ subimage. (al)-(a3) The EL subimages. (b1)-(b3) The results of FIR method with $K_{\Delta f}=0.5$. (c1)-(c3) The results of FIR method with $K_{\Delta f}=1$. (d1)-(d3) The results of FIR method with $K_{\Delta f}=1.5$. (e1)-(e3) The results of our proposed method. The black regions and lines indicate detected defects.

$1024 \times 1024$ pixels with 12-bit gray levels. Most researches on solar cells extrinsic defect detection focus on microcrack detection and receive valuable success. However, there are few researches working on finger interruption detection. Up to date, we can only find one research article working on finger interruption detection. Tsai et al. [6] proposed to detect finger interruption by using Fourier image reconstruction (FIR). Thus we will compare our proposed method with FIR method. In the FIR method, an EL image needs to be divided to several subimages for detecting defects. The size of subimage is suggested to between $55 \times 55$ and $95 \times 95$. In our experiments, we will compare our proposed method with $55 \times 55,75 \times 75$, and $95 \times 95$ subimages of the FIR method and show the experimental results in binary images. The threshold parameters $k_{\Delta f}$ of the FIR method are set to $0.5,1$, and 1.5 in each subimage size.

The results of detecting EL images with noninterrupted finger defects are shown in Figures 7, 8, and 9 with subimage 


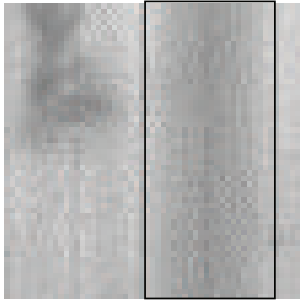

(a1)

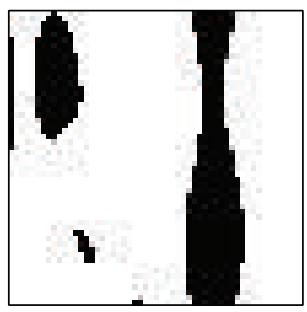

(b1)

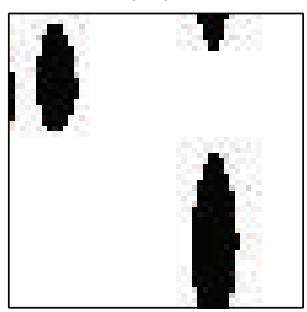

(c1)

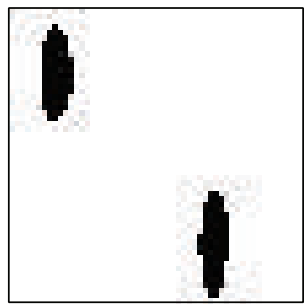

(d1)

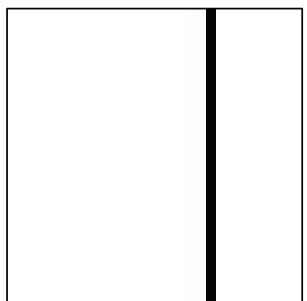

(e1)

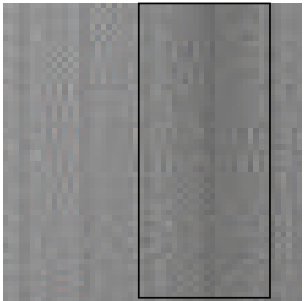

(a2)

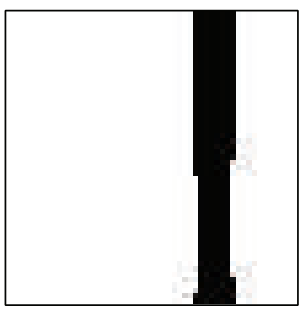

(b2)

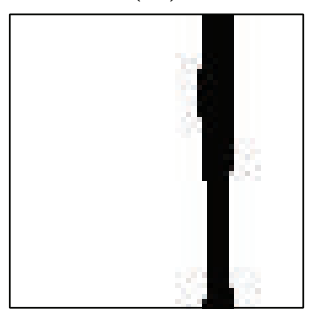

(c2)

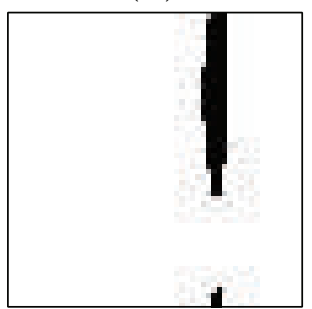

(d2)

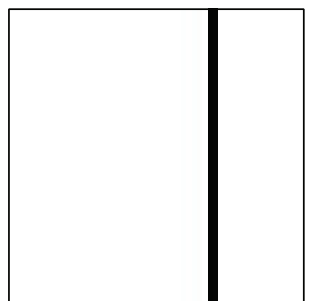

(e2)

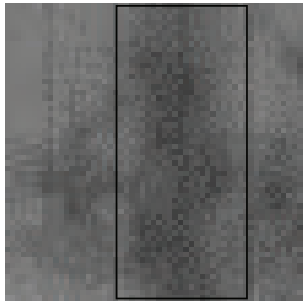

(a3)

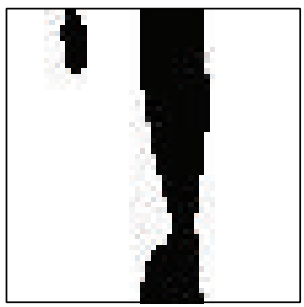

(b3)

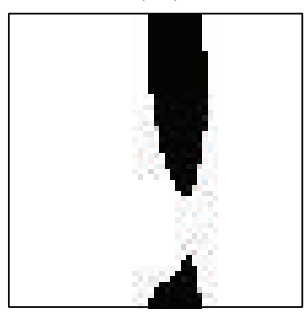

(c3)

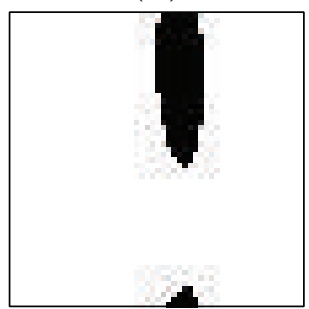

(d3)

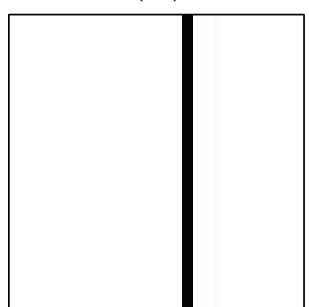

(e3)

FIGURE 10: The experimental results of EL subimages with an interrupted finger defect on a $55 \times 55$ subimage. (a1)-(a3) The EL subimages; the solid rectangle indicates the area of interrupted finger defect. (b1)-(b3) The results of FIR method with $K_{\Delta f}=0.5$. (c1)-(c3) The results of FIR method with $K_{\Delta f}=1$. (d1)-(d3) The results of FIR method with $K_{\Delta f}=1.5$. (e1)-(e3) The results of our proposed method. The black regions and lines indicate detected defects.

size $55 \times 55,75 \times 75$, and $95 \times 95$, respectively. The threshold parameter $k_{\Delta f}$ in the FIR method is set to $0.5,1$, and 1.5 for comparison. In Figures 7 to 9, the FIR method tends to misjudge stripe noise (intrinsic defects) as extrinsic defects, as shown in (b1-b3), (c1-c3), and (d1-d3). Our proposed method will misjudge noise as interrupted finger only when the characteristic of noise strip is similar to interrupted finger, as shown in Figures 7(e3), 8(e3), and 9(e3).
The results of detecting EL images with interrupted finger defect are shown in Figures 10, 11, and 12 with subimage size $55 \times 55,75 \times 75$, and $95 \times 95$, respectively. The threshold parameter $k_{\Delta f}$ in the FIR method is set to $0.5,1$, and 1.5 for comparison. In Figures 10, 11, and 12, the results of FIR method highly depended on the setting of threshold $k_{\Delta f}$. If $k_{\Delta f}$ is too small, the FIR method tends to misjudge noises as interrupted fingers; if $k_{\Delta f}$ is too large, the FIR method tends 


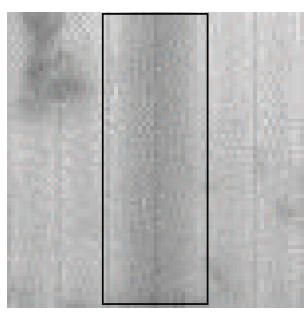

(a1)

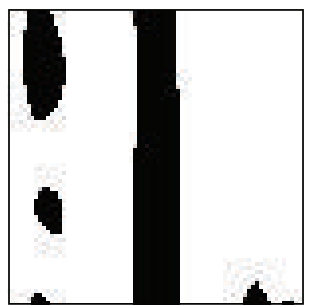

(b1)

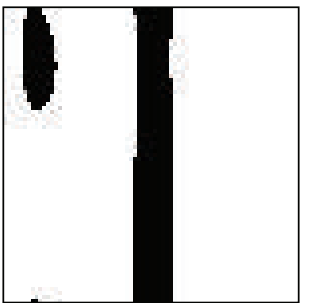

(c1)

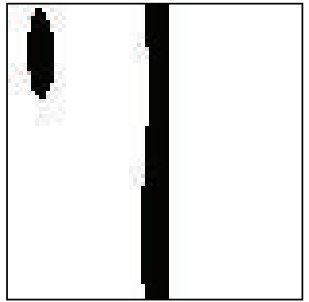

(d1)

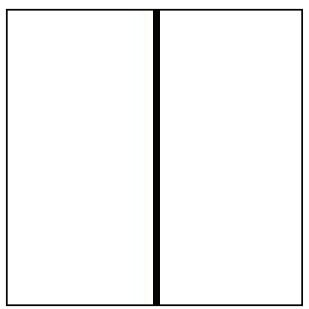

(e1)

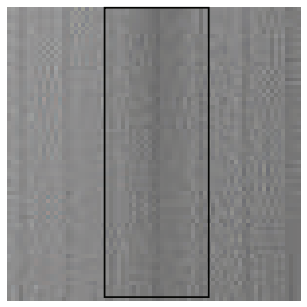

(a2)

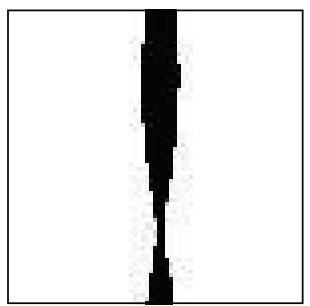

(b2)

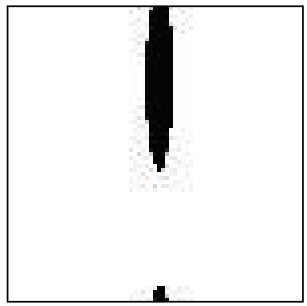

(c2)

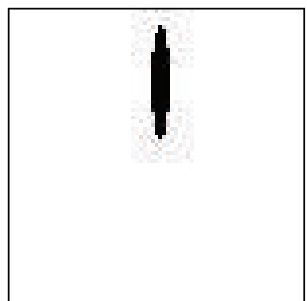

(d2)

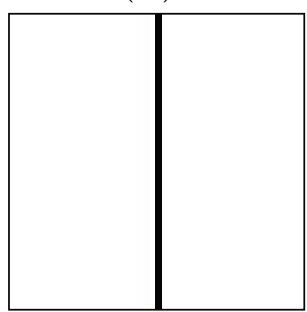

(e2)

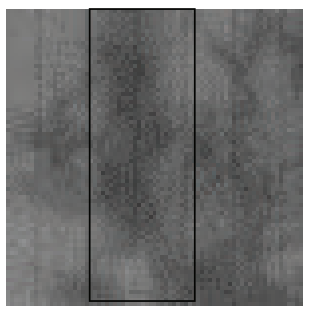

(a3)

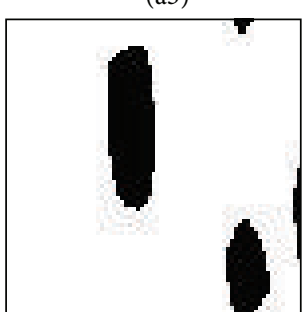

(b3)

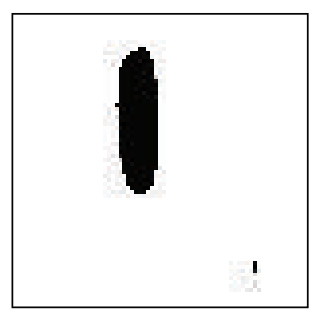

(c3)

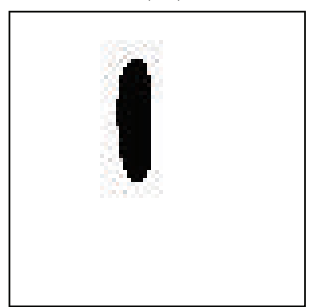

(d3)

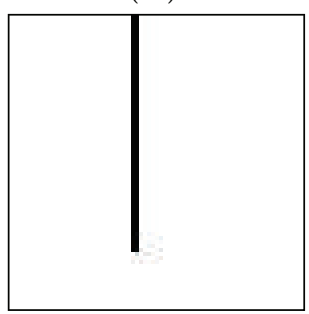

(e3)

FIGURE 11: The experimental results of EL subimages with an interrupted finger defect on a $75 \times 75$ subimage. (a1)-(a3) The EL subimages; the solid rectangle indicates the area of interrupted finger defect. (b1)-(b3) The results of FIR method with $K_{\Delta f}=0.5$. (c1) $-(\mathrm{c} 3)$ The results of FIR method with $K_{\Delta f}=1$. (d1)-(d3) The results of FIR method with $K_{\Delta f}=1.5$. (e1)-(e3) The results of our proposed method. The black regions and lines indicate detected defects.

to ignore suspected areas, even if they are interrupted fingers. Our proposed method can find almost all interrupted fingers, as shown in Figures 10(el-e3), 11(el-e3), and 12(el-e3).

Actually, our proposed method needs not to divide the EL image to subimages. The above analysis is for comparison purpose only. In our proposed method, we locate 4 ROIs in an EL image, as shown in Figure 13(a). The detection results are shown in Figure 13(b). In Figure 13(a), the interrupted fingers are marked in white rectangles. In Figure 13(b), the solid rectangles indicate the interrupted fingers found. By our method, the dashed rectangles indicate the interrupted finger missed. The miss detection is due to the fact that the interrupted ratio of a finger is smaller than our predefined ratio. The four ROIs are numbered as 1 to 4 from top to bottom. Since the heights of the 1st and 4th ROIs are similar, the heights of the 2nd and 3rd ROIs are similar; their accuracy rates are slightly different, as shown in Table 1 . In Table 1, the accuracy rate, miss rate, and false alarm rate are 


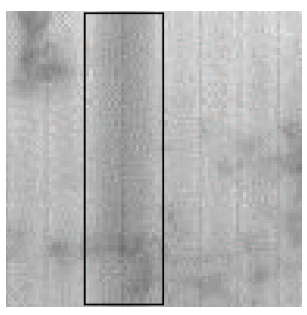

(a1)

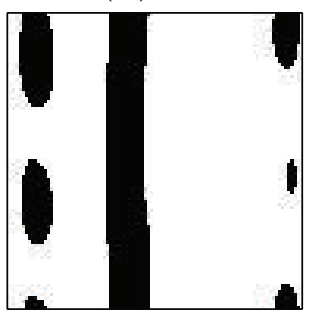

(b1)

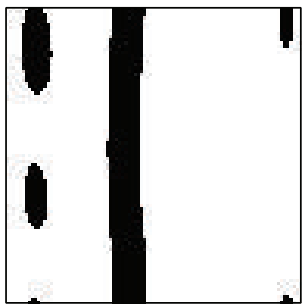

(c1)

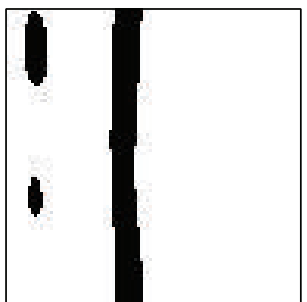

(d1)

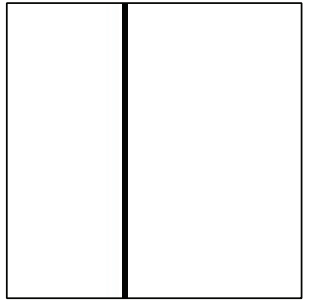

(e1)

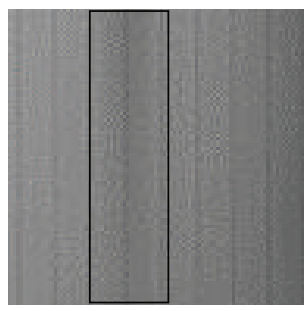

(a2)

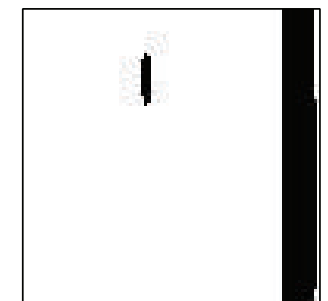

(b2)

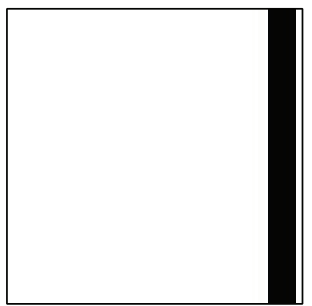

(c2)

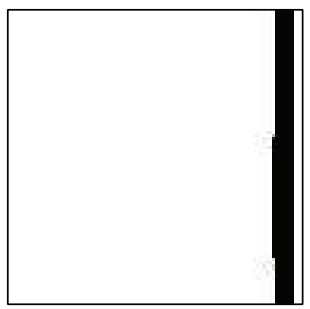

(d2)

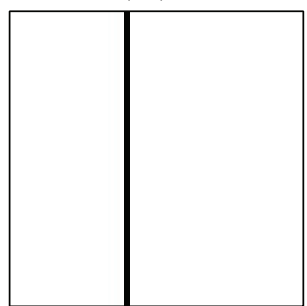

(e2)

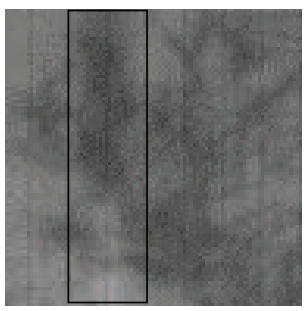

(a3)

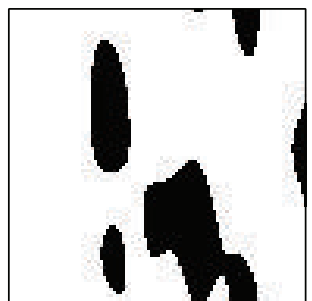

(b3)

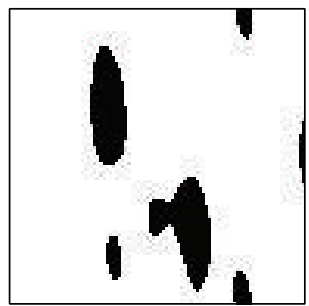

(c3)

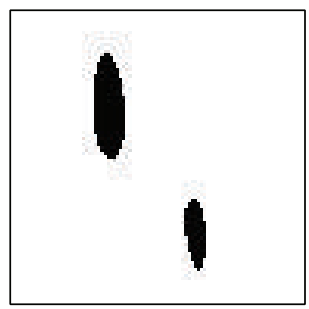

(d3)

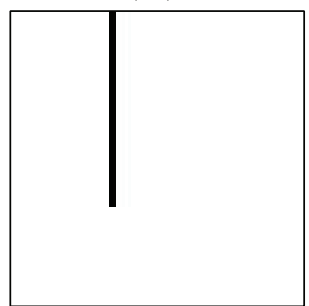

(e3)

FIGURE 12: The experimental results of EL subimages with an interrupted finger defect on a $95 \times 95$ subimage. (a1)-(a3) The EL subimages; the solid rectangle indicates the area of interrupted finger defect. (b1)-(b3) The results of FIR method with $K_{\Delta f}=0.5$. (c1)-(c3) The results of FIR method with $K_{\Delta f}=1$. (d1)-(d3) The results of FIR method with $K_{\Delta f}=1.5$. (e1)-(e3) The results of our proposed method. The black regions and lines indicate detected defects.

calculated by

$$
\begin{gathered}
\text { Accuracy rate }=\frac{\text { True positive }+ \text { True negative }}{\text { True positive }+ \text { True negative }+ \text { False positive }+ \text { False negative }}, \\
\text { Miss rate }=\frac{\text { False negative }}{\text { True positive }+ \text { False negative }}, \\
\text { False alarm rate }=\frac{\text { False positive }}{\text { True negative }+ \text { False positive }} .
\end{gathered}
$$



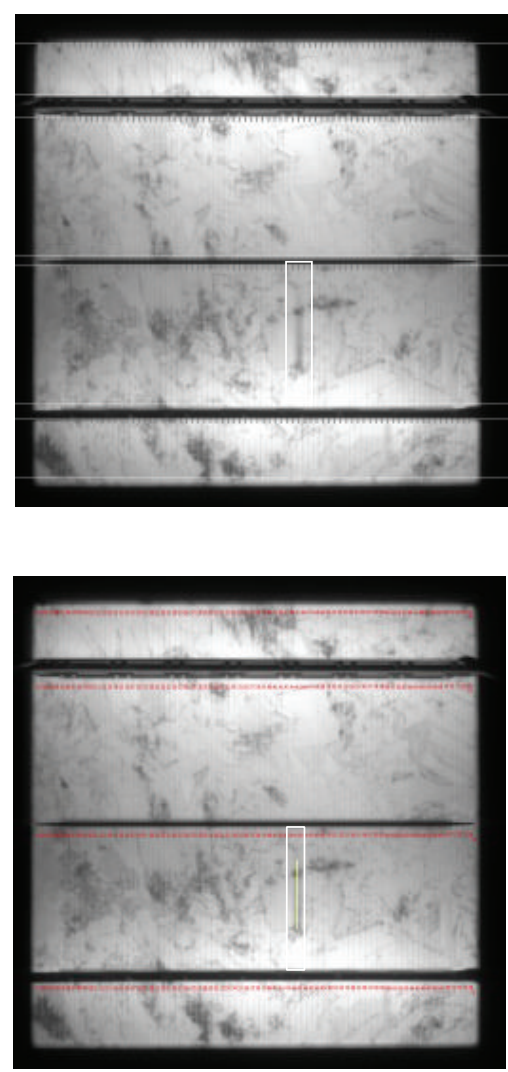
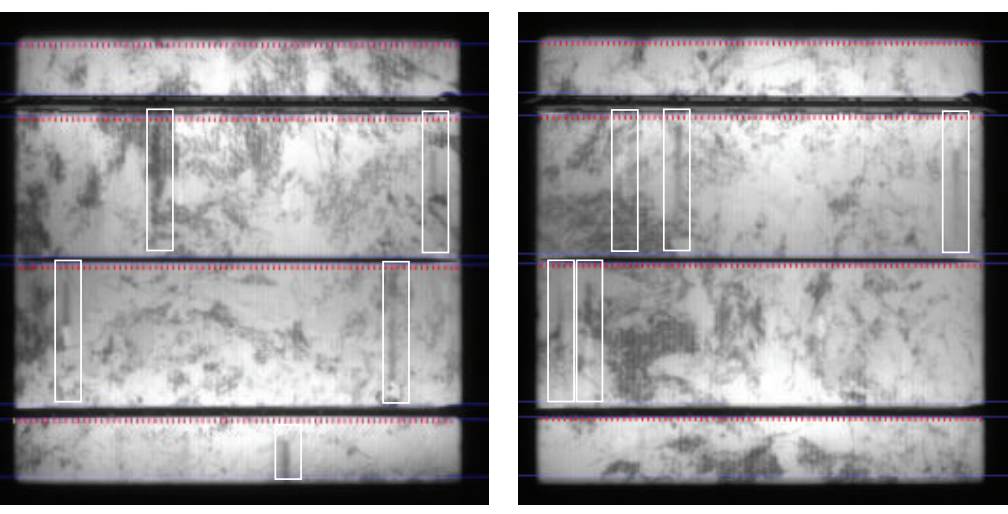

(a)
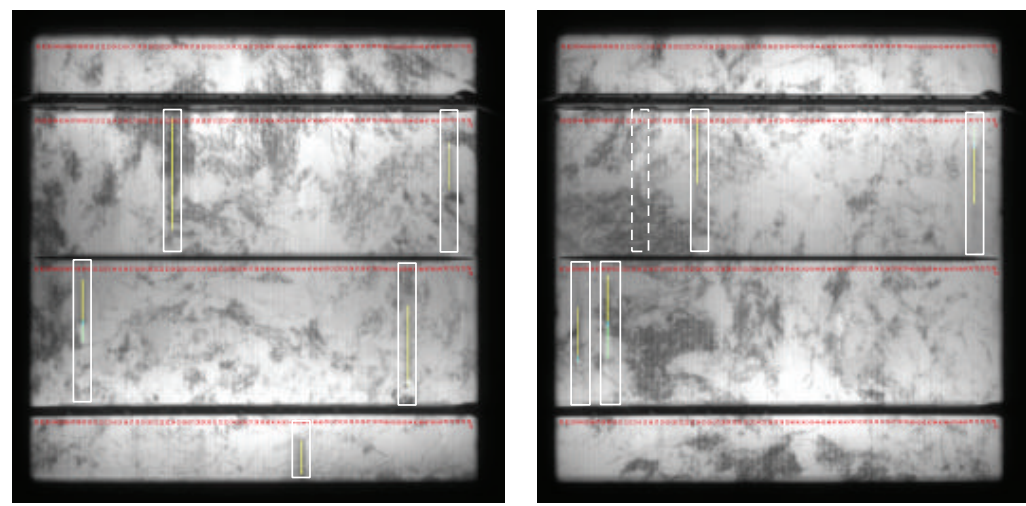

(b)

FiguRE 13: Solar cells in our experiments. (a) The EL images; the solid rectangles indicate the interrupted finger defects. (b) The detection results of (a); the solid rectangles indicate the interrupted finger defects found and the dashed rectangles indicate the interrupted finger defects missed.

TABLE 1: The statistics of detection results.

\begin{tabular}{lcc}
\hline & 1st and 4nd ROI & 2nd and 3rd ROI \\
\hline Accuracy rate & $99.07 \%$ & $99.58 \%$ \\
False negative rate & $6.89 \%$ & $2.39 \%$ \\
False positive rate & $0.66 \%$ & $0.34 \%$ \\
\hline
\end{tabular}

\section{Conclusions}

In this paper, we have proposed a supervised classification method to detect interrupted finger defects in multicrystalline solar cells. The interrupted fingers cannot be visually observed in an image with the conventional CCD imaging system. The electroluminescence (EL) imaging technique is thus used to highlight the interrupted fingers in the sensed image. The automatic extrinsic defect detection in EL images of multicrystalline silicon solar cell is usually very difficult due to the disturbances of intrinsic defects. The proposed method can extract essential features from fingers and efficiently recognize defects. The extracted features come from the variation of gray-level on both sides of fingers; these features reflect the property of defects better than shape features. The proposed method is more flexible than method of setting threshold values.

We have applied the proposed method in detecting defects on a variety of solar cells. Experimental results show that the proposed method achieves superior results over the Fourier image reconstruction method in the situations of background interference and unobvious defects. Moreover, our proposed method can quickly locate the ROIs for detection. In the fix-sized subimage method, a defect may be divided into different subimages and thus affect the detection result. Compared to fix-sized subimage method, the proposed method can detect the defects without concerning the size of subimages and eventually get a better detection result.

\section{Conflict of Interests}

The authors declare that there is no conflict of interests regarding the publication of this paper. 


\section{References}

[1] C. Sener and V. Fthenakis, "Energy policy and financing options to achieve solar energy grid penetration targets: accounting for external costs," Renewable and Sustainable Energy Reviews, vol. 32, pp. 854-868, 2014.

[2] M. Abdelhamid, R. Singh, and M. Omar, "Review of microcrack detection techniques for silicon solar cells," IEEE Journal of Photovoltaics, vol. 4, no. 1, pp. 514-524, 2014.

[3] P. Chaturvedi, B. Hoex, and T. M. Walsh, "Broken metal fingers in silicon wafer solar cells and PV modules," Solar Energy Materials and Solar Cells, vol. 108, pp. 78-81, 2013.

[4] R. De Rose, A. Malomo, P. Magnone et al., "A methodology to account for the finger interruptions in solar cell performance," Microelectronics Reliability, vol. 52, no. 9-10, pp. 2500-2503, 2012.

[5] T. Fuyuki and A. Kitiyanan, "Photographic diagnosis of crystalline silicon solar cells utilizing electroluminescence," Applied Physics A: Materials Science and Processing, vol. 96, no. 1, pp. 189-196, 2009.

[6] D.-M. Tsai, S.-C. Wu, and W.-C. Li, "Defect detection of solar cells in electroluminescence images using Fourier image reconstruction," Solar Energy Materials and Solar Cells, vol. 99, pp. 250-262, 2012.

[7] M. Israil, S. A. Anwar, and M. Z. Abdullah, "Automatic detection of micro-crack in solar wafers and cells: a review," Transactions of the Institute of Measurement and Control, vol. 35, no. 5, pp. 606-618, 2013.

[8] Z. Wang, F. Yang, G. Pan, J. Gao, and H. Zhang, "Research on detection technology for solar cells multi-defects in complicated background," Journal of Information and Computational Science, vol. 11, no. 2, pp. 449-459, 2014.

[9] D.-M. Tsai, S.-C. Wu, and W.-Y. Chiu, "Defect detection in solar modules using ICA basis images," IEEE Transactions on Industrial Informatics, vol. 9, no. 1, pp. 122-131, 2013.

[10] M. Filippone, F. Camastra, F. Masulli, and S. Rovetta, "A survey of kernel and spectral methods for clustering," Pattern Recognition, vol. 41, no. 1, pp. 176-190, 2008.

[11] J. Shi and J. Malik, "Normalized cuts and image segmentation," IEEE Transactions on Pattern Analysis and Machine Intelligence, vol. 22, no. 8, pp. 888-905, 2000.

[12] W.-Y. Chen, Y. Song, H. Bai, C.-J. Lin, and E. Y. Chang, "Parallel spectral clustering in distributed systems," IEEE Transactions on Pattern Analysis and Machine Intelligence, vol. 33, no. 3, pp. 568586, 2011.

[13] U. von Luxburg, "A tutorial on spectral clustering," Statistics and Computing, vol. 17, no. 4, pp. 395-416, 2007.

[14] L. J. O’Donnell and C.-F. Westin, "Automatic tractography segmentation using a high-dimensional white matter atlas," IEEE Transactions on Medical Imaging, vol. 26, no. 11, pp. 1562-1575, 2007. 


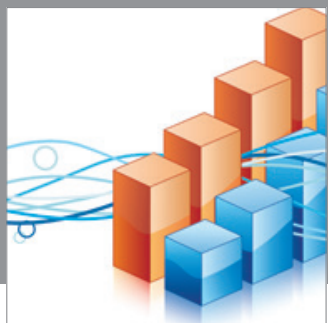

Advances in

Operations Research

mansans

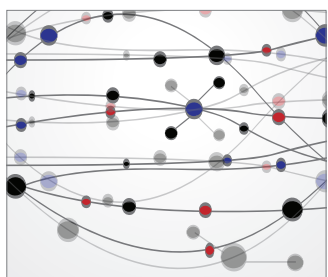

The Scientific World Journal
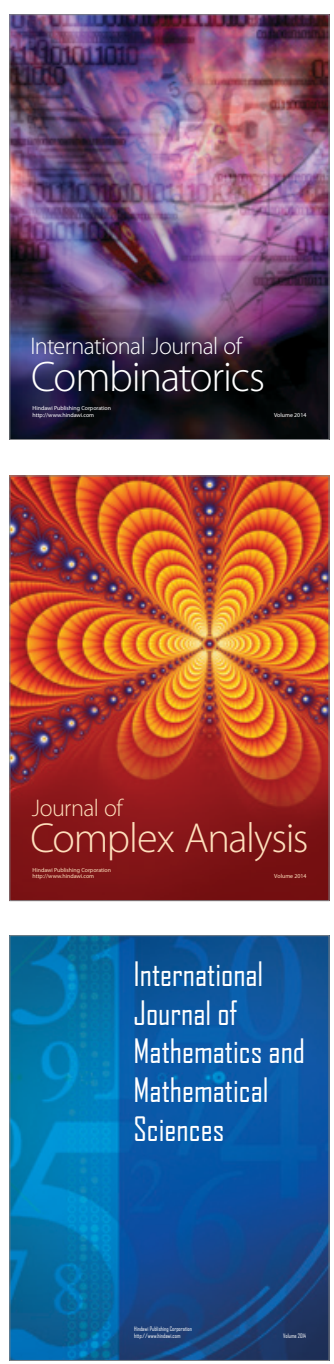
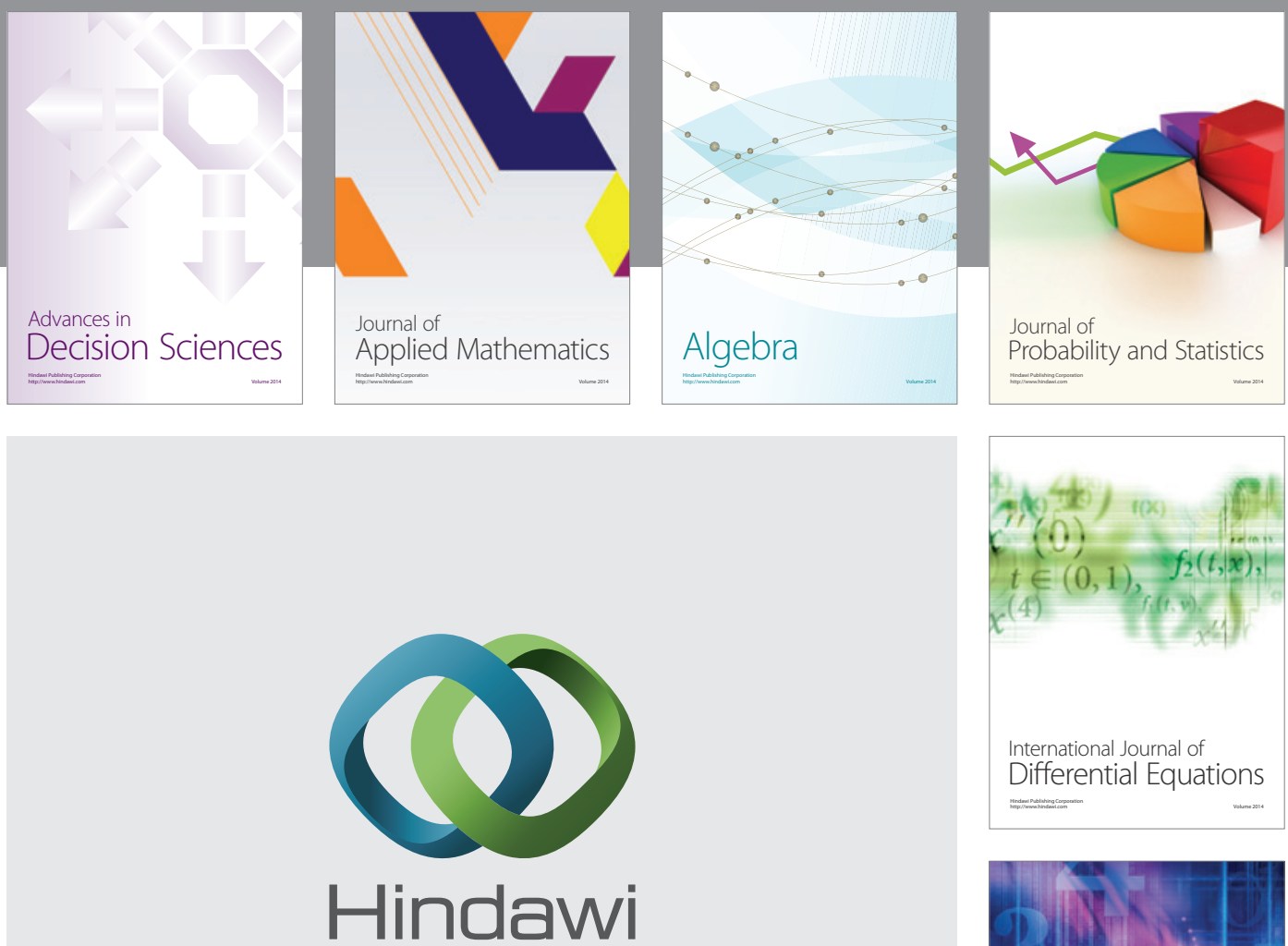

Submit your manuscripts at http://www.hindawi.com
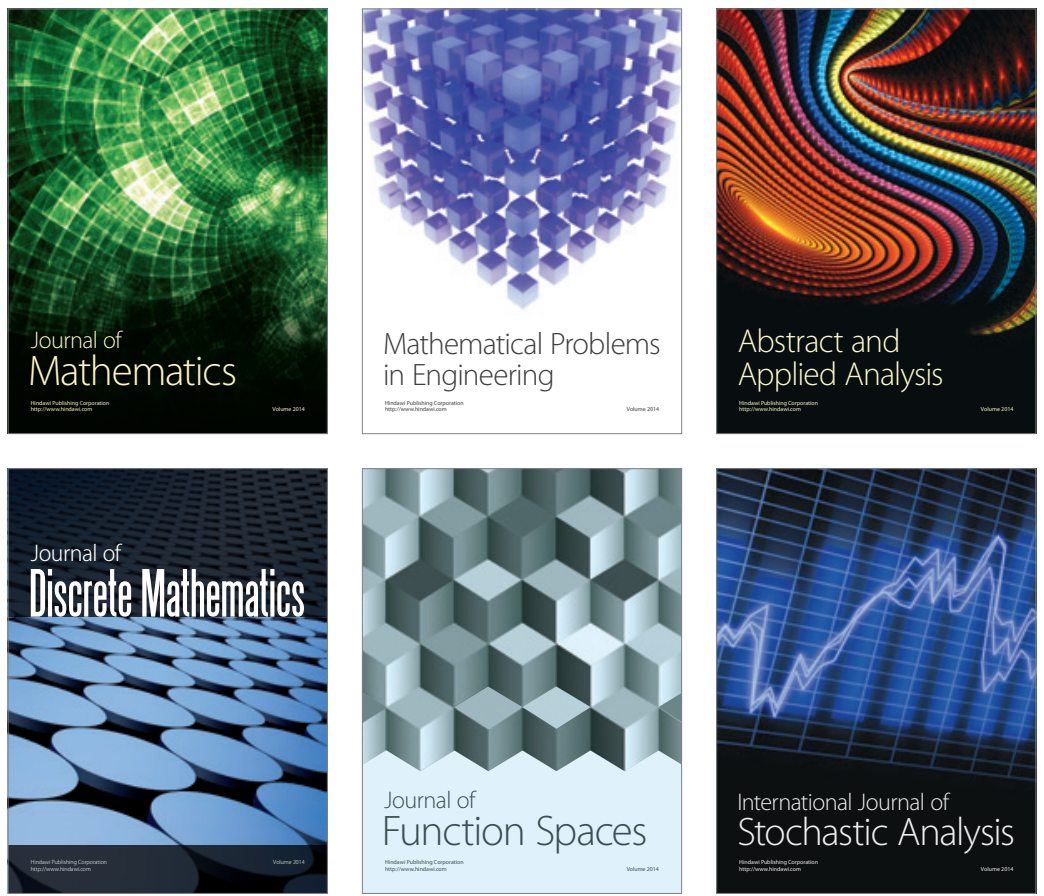

Journal of

Function Spaces

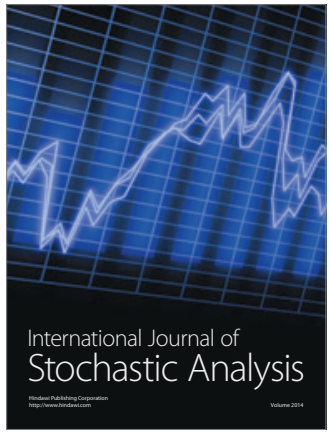

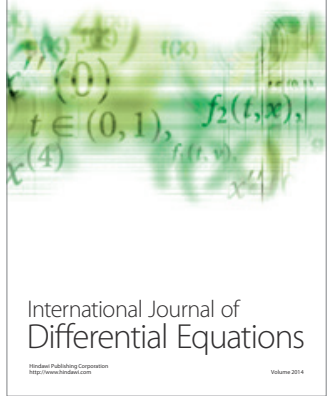
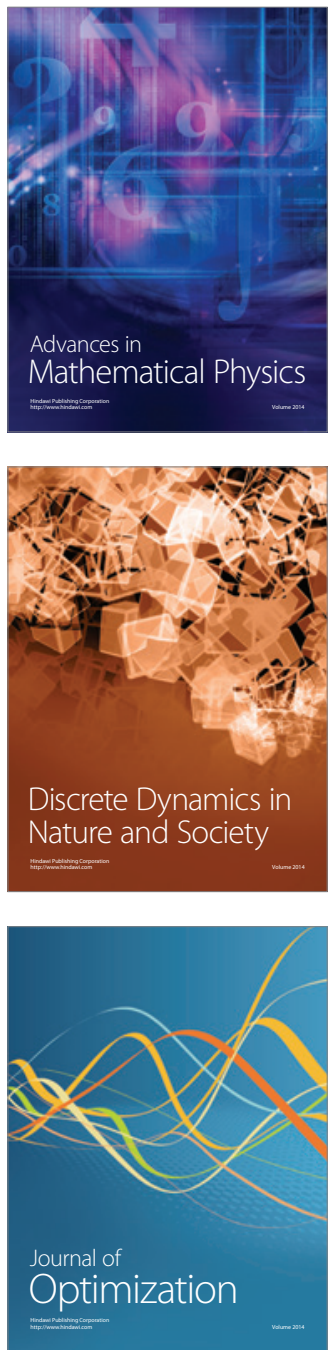